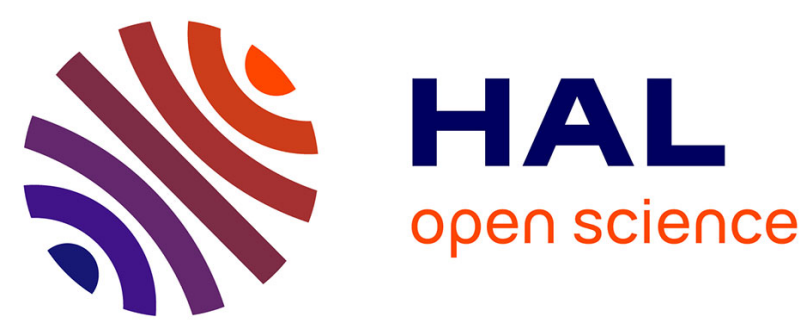

\title{
A Structural Theory of Rhythm Notation based on Tree Representations and Term Rewriting
}

Florent Jacquemard, Pierre Donat-Bouillud, Jean Bresson

\section{To cite this version:}

Florent Jacquemard, Pierre Donat-Bouillud, Jean Bresson. A Structural Theory of Rhythm Notation based on Tree Representations and Term Rewriting. Mathematics and Computation in Music: 5th International Conference, MCM 2015, Oscar Bandtlow and Elaine Chew, Jun 2015, London, United Kingdom. pp.12. hal-01138642

\section{HAL Id: hal-01138642 \\ https://hal.inria.fr/hal-01138642}

Submitted on 2 Apr 2015

HAL is a multi-disciplinary open access archive for the deposit and dissemination of scientific research documents, whether they are published or not. The documents may come from teaching and research institutions in France or abroad, or from public or private research centers.
L'archive ouverte pluridisciplinaire HAL, est destinée au dépôt et à la diffusion de documents scientifiques de niveau recherche, publiés ou non, émanant des établissements d'enseignement et de recherche français ou étrangers, des laboratoires publics ou privés. 


\title{
A Structural Theory of Rhythm Notation based on Tree Representations and Term Rewriting *
}

\author{
Florent Jacquemard ${ }^{1}$, Pierre Donat-Bouillud ${ }^{1,2}$, and Jean Bresson ${ }^{1}$ \\ ${ }^{1}$ UMR STMS: IRCAM-CNRS-UPMC and INRIA, Paris, France. \\ florent. jacquemard@inria.fr, jean.bresson@ircam.fr \\ 2 ENS Rennes, Ker Lann Campus, France. \\ pierre.donat-bouillud@ens-rennes.fr
}

\begin{abstract}
We present a tree-based symbolic representation of rhythm notation suitable for processing with purely syntactic theoretical tools such as term rewriting systems or tree automata. Then we propose an equational theory, defined as a set of rewrite rules for transforming these representations. This theory is complete in the sense that from a given rhythm notation the rules permit to generate all notations of equivalent durations.
\end{abstract}

\section{Introduction}

Term Rewriting Systems (TRSs) [8] are well established formalisms for tree processing (transformation and reasoning). With solid theoretical foundations, they are used in a wide range of applications, to name a few: automated reasoning, natural language processing, foundations of Web data, etc. TRSs perform in-place transformations in trees by the replacement of patterns, as defined by oriented equations called rewrite rules. They are a classical model for symbolic computation, used for rule-based modeling, simulation and verification of complex systems or software (see e.g. the languages $\mathrm{TOM}^{3}$ and Maude ${ }^{4}$ ). Tree Automata (TAs) [7] are finite state recognizers of trees which permit to characterize specific types of tree-structured data (regular tree languages). They are often used in conjunction with TRSs, acting as filters in the explorations of sets of trees computed by rewriting.

It is also common to use trees to represent hierarchical structures in symbolic music (see [15] for a survey). For instance, the GTTM [13] uses trees to analyse inner relations in musical pieces. Trees are also a natural representation of rhythms, where durations are expressed as a hierarchy of subdivisions. Computer-aided composition (CAC) environments such as Patchwork and OpenMusic $[3,6]$ use structures called rhythm trees (RTs) for representing and programming rhythms [2]. Such hierarchical, notation-oriented approach (see also

\footnotetext{
* This work is part of the EFFICACe project funded by the French National Research Agency (ANR-13-JS02-0004-01). A more complete version of this paper is available at https://hal.inria.fr/hal-01134096/.

${ }^{3}$ http://tom.loria.fr

${ }^{4}$ http://maude.cs.illinois.edu
} 
[15]) is complementary to the performance-oriented formats corresponding to the MIDI notes' onsets and offsets in standard computer music systems. It also provides a more structured representation of time than music notation formats such as MusicXML [9] or Guido [12], where durations are expressed with integer values. As highly structured representations, trees enable powerful manipulation and generation processes in the rhythmic domain (see for instance [11]), and enforce some structural constraints on duration sequences.

In this paper, we propose a tree-structured representation of rhythm suitable for defining a set of rewriting rules (i.e. oriented equations) preserving rhythms, while allowing simplifications of notation. This representation bridges CAC rhythm structures with formal tree-processing approaches, and enables a number of new manipulations and applications in both domains. In particular, rewriting rules can be seen as an axiomatization of rhythm notation, which can be applied to reasoning on equivalent notations in computer-aided music composition or analysis.

\section{Preliminary Definitions}

Let us assume given a countable set of variables $\mathcal{X}$, and a ranked signature $\Sigma$ which is a finite set of symbols, each symbol being assigned a fixed arity. We denote as $\Sigma_{p}$ the subset of $\Sigma$ of symbols of arity $p$.

Trees. A $\Sigma$-labelled tree $t$ (called tree for short in the rest of the paper) is either a single node, called root of $t$ and denoted by root $(t)$, labeled with a variable $x \in \mathcal{X}$ or one constant symbol of $a_{0} \in \Sigma_{0}$, or it is made of one node also denoted by $\operatorname{root}(t)$ and labeled with a symbol $a \in \Sigma_{n}(n>0)$, and of an ordered sequence of $n$ direct subtrees $t_{1}, \ldots, t_{n}$.

In the first case, the tree $t$ is simply denoted $x$ or $a_{0}$. In the second case, $t$ is denoted $a\left(t_{1}, \ldots, t_{n}\right), \operatorname{root}(t)$ is called the parent of respectively $\operatorname{root}\left(t_{1}\right), \ldots, \operatorname{root}\left(t_{n}\right)$, and the latter are called children of $\operatorname{root}(t)$. Moreover, for all $i, 1<i \leq n$, $\operatorname{root}\left(t_{i-1}\right)$ is called the previous sibling of $\operatorname{root}\left(t_{i}\right)$. For $1 \leq i \leq p$, the previous cousin of $\operatorname{root}\left(t_{i}\right)$ is either $\operatorname{root}\left(t_{i-1}\right)$ if $i>1$, or the last children of the previous cousin of the parent $\operatorname{root}(t)$ if $i=1$ and if this node exists. In other terms, the previous cousin of a node $\nu$ in a tree $t$ is the node immediately at the left of $\nu$ in $t$, at the same level. A node in a tree $t$ with no children is called a leaf of $t$. In the following, we will consider the sequence of leaves of a tree $t$ as enumerated by a depth-first-search $(d f s)$ traversal.

Example 1. Some trees are depicted in Figures 1 to 5 . In the tree 2(n, 3(o, n, n) ) of Figure 2(b), the first leaf in $d f s$ ordering (labeled with $\mathrm{n}$ ) is the previous cousin of the node labeled by 3 , and the second leaf in $d f s$ ordering (labeled with o) is the previous sibling of the third leaf (labeled with $n$ ), which is in turn the previous sibling of the fourth leaf (also labeled with $\mathrm{n}$ ).

The set of trees built over $\Sigma$ and $\mathcal{X}$ is denoted $\mathcal{T}(\Sigma, \mathcal{X})$, and the subset of trees without variables $\mathcal{T}(\Sigma)$. The definition domain of a tree $t \in \mathcal{T}(\Sigma, \mathcal{X})$, 


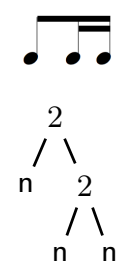

(a) $\frac{1}{2} \frac{1}{4} \frac{1}{4}$
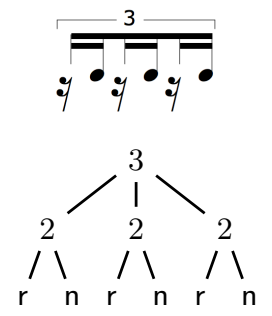

(b) $\left[\frac{1}{6}\right] \frac{1}{6}\left[\frac{1}{6}\right] \frac{1}{6}\left[\frac{1}{6}\right] \frac{1}{6}$
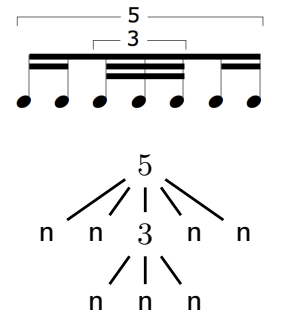

(c) $\frac{1}{5} \frac{1}{5} \frac{1}{15} \frac{1}{15} \frac{1}{15} \frac{1}{5} \frac{1}{5}$

Fig. 1. Simple trees of $\mathcal{T}\left(\Sigma_{\mathrm{rn}}\right)$ with their corresponding rhythmic notations and values.

denoted by $\operatorname{dom}(t)$, is the set of nodes of $t$. The size $|t|$ of $t$ is the cardinality of $\operatorname{dom}(t)$. Given $\nu \in \operatorname{dom}(t): t(\nu) \in \Sigma \cup \mathcal{X}$ is the label of $\nu$ in $t,\left.t\right|_{\nu}$ is the subtree of $t$ at node $\nu, t\left[t^{\prime}\right]_{\nu}$ is the tree obtained from $t$ by replacement of $\left.t\right|_{\nu}$ by $t^{\prime}$. We define the depth of a single-node tree $x$ or $a_{0}$ as 0 and the depth of $a\left(t_{1}, \ldots, t_{n}\right)$ as $1+$ the maximal depth of $t_{1}, \ldots, t_{n}$.

Pattern Matching. We call pattern over $\Sigma$ a finite sequence of trees of $\mathcal{T}(\Sigma, \mathcal{X})$ of length $n \geq 1$, denoted as $t_{1} ; \ldots ; t_{n}$ (the symbol ; denotes the cousin relation). The size of a pattern is the sum of the sizes of its constituting trees.

A substitution is a mapping from variables of $\mathcal{X}$ into trees of $\mathcal{T}(\Sigma, \mathcal{X})$ with a finite domain. The application of substitutions is homomorphically extended from variables to trees and patterns: $\sigma\left(a\left(t_{1}, \ldots, t_{p}\right)\right)=a\left(\sigma\left(t_{1}\right), \ldots, \sigma\left(t_{p}\right)\right)$ and $\sigma\left(t_{1} ; \ldots ; t_{n}\right)=\sigma\left(t_{1}\right) ; \ldots ; \sigma\left(t_{n}\right)$.

A tree $t$ matches a pattern $t_{1} ; \ldots ; t_{n}$ at node $\nu$ with substitution $\sigma$ if there exists a sequence of successive cousins $\nu_{1}, \ldots, \nu_{n}$ in $\operatorname{dom}(t)$ such that $\nu_{1}=\nu$ and $\left.t\right|_{\nu_{i}}=\sigma\left(t_{i}\right)$ for all $1 \leq i \leq n$. When there exists such a sequence of cousins, we write $t\left[t_{1}^{\prime} ; \ldots ; t_{n}^{\prime}\right]_{\nu}$ for the iterated replacement $t\left[t_{1}^{\prime}\right]_{\nu_{1}} \ldots\left[t_{n}^{\prime}\right]_{\nu_{n}}$.

Term Rewriting. A rewrite rule is a pair of patterns of same length denoted $\ell_{1} ; \ldots ; \ell_{n} \rightarrow r_{1} ; \ldots ; r_{n}$ and a tree rewrite system $(T R S)$ over $\Sigma$ is a finite set of rewrite rules over $\Sigma$.

A tree $s \in \mathcal{T}(\Sigma, \mathcal{X})$ rewrites to $t \in \mathcal{T}(\Sigma, \mathcal{X})$ with a TRS $\mathcal{R}$ over $\Sigma$, denoted by $s \underset{\mathcal{R}}{\longrightarrow}(\mathcal{R}$ may be omitted when clear from context) if there exists a rewrite rule $\ell_{1} ; \ldots ; \ell_{n} \rightarrow r_{1} ; \ldots ; r_{n} \in \mathcal{R}$, a node $\nu \in \operatorname{dom}(s)$ and a substitution $\sigma$ over $\Sigma$ such that $s$ matches $\ell_{1} ; \ldots ; \ell_{n}$ at $\nu$ with $\sigma$ and $t=s\left[\sigma\left(r_{1}\right) ; \ldots ; \sigma\left(r_{n}\right)\right]_{\nu}$. The reflexive and transitive closure of $\overrightarrow{\mathcal{R}}$ is denoted by $\underset{\mathcal{R}}{\mathcal{R}}$, and the reflexive, symmetric and transitive closure by $\underset{\mathcal{R}}{*}$.

This definition strictly generalizes the standard notion of term rewriting [8], which corresponds to the particular case of rewrite rules with patterns of length one (i.e. trees). Our notion of rewriting cousin-patterns can be captured by extensions of rewriting such as the spatial programming language MGS [5]. 


\section{Ranked Tree Representation of Rhythm Notation}

We consider a particular signature $\Sigma_{\mathrm{rn}}$ for expressing rhythm notations. It contains the following symbols of arity zero (constant symbols): $\mathrm{n}$ (representing a note), $r$ (rest), s (slur), d (dot), and o (for composition of durations, as explained below). Moreover, $\Sigma_{\mathrm{rn}}$ contains a subset $\mathbb{P}$ of symbols denoted as prime integers, each $p \in \mathbb{P}$ having arity $p$, and a copy $\overline{\mathbb{P}}=\{\bar{p} \mid p \in \mathbb{P}\}$, where $\bar{p}$ has also arity $p$. More precisely, $\mathbb{P}$ is assumed to contain a (small) prime integer $\max (\mathbb{P})$, assumed fixed throughout the paper, and all prime numbers smaller than $\max (\mathbb{P})$, i.e. $\mathbb{P}=\{2,3,5, \ldots, \max (\mathbb{P})\}$. Typically, $\max (\mathbb{P})=11$. The symbols of $\mathbb{P} \cup \overline{\mathbb{P}}$ will be used to build tuplets defined by equal subdivision of a duration.

\subsection{Tree Semantics}

Intuitively, a tree of $\mathcal{T}\left(\Sigma_{\mathrm{rn}}\right)$ represents a sequence of notes and rests, denoted by symbols $\mathrm{n}$ and $\mathrm{r}$ in the leaves, their duration being encoded in the structure of the tree. The symbols $s$ and $d$ are used to group the durations of successive leaves. The symbol o is used to group the durations of successive cousins, and possibly further subdivise the summed duration.

Formally, given a tree $t \in \mathcal{T}\left(\Sigma_{\mathrm{rn}}, \mathcal{X}\right)$, we associate recursively a duration value, denoted $\operatorname{dur}_{t}(\nu)$, to each node $\nu \in \operatorname{dom}(t)$ as follows:

- If $\nu=\operatorname{root}(t)$, then $\operatorname{dur}_{t}(\nu)$ is a number of beats $n \geq 1$ associated to $t(t$ can represent e.g. one or several beats or a whole bar).

- Otherwise, let $\nu_{0}$ be the parent of $\nu$ in $t$ and let $p$ be the arity of $t\left(\nu_{0}\right)$, $d u r_{t}(\nu)=\frac{d u r_{t}\left(\nu_{0}\right)}{p}+c d u r_{t}(\nu)$, where $c d u r_{t}(\nu)=d u r_{t}\left(\nu^{\prime}\right)$ if $\nu$ has a previous cousin $\nu^{\prime}$ such that $t\left(\nu^{\prime}\right)=0, c d u r_{t}(\nu)=0$ otherwise.

A tree $t \in \mathcal{T}\left(\Sigma_{\mathrm{rn}}\right) \backslash\{\mathrm{o}\}$ represents a sequence $\operatorname{val}(t)$ of durations. Let $k \geq 1$ be the number of leaves of $t$ not labeled by $o$ and let $\nu_{1} \ldots, \nu_{k}$ be the enumeration of these leaves in $d f s$. The duration sequence of $t$ is defined as $d s(t)=$ $\left\langle t\left(\nu_{1}\right), \operatorname{dur}_{t}\left(\nu_{1}\right)\right\rangle, \ldots,\left\langle t\left(\nu_{k}\right), d u r_{t}\left(\nu_{k}\right)\right\rangle$. Let $i_{1}, \ldots, i_{\ell+1}(\ell \geq 0)$ be an increasing sequence of indices defined as follows: $i_{1}=1, i_{2}, \ldots, i_{\ell}$ is the subsequence of indices of nodes in $\left\{\nu_{2} \ldots, \nu_{k}\right\}$ labeled by $\mathrm{n}$ or $\mathrm{r}, i_{\ell+1}=k+1$.

The rhythmic value val $(t)$ of $t$ is the sequence of pairs $u_{1}, \ldots, u_{\ell}$, where for each $j, 1 \leq j \leq \ell$,

$$
u_{j}=\left\langle t\left(\nu_{i_{j}}\right), \sum_{i=i_{j}}^{i=i_{j+1}-1} d u r_{t}\left(\nu_{i}\right)\right\rangle
$$

According to this definition, the first component of each pair $u_{j}$ is either $\mathrm{n}$ or $\mathrm{r}$, and the second component is the sum of the durations of next leaves labeled by $s$ or d. For convenience, we shall omit the first components of pairs, and denote $\operatorname{val}(t)$ as a sequence of durations, where the duration of rests $r$ are written in brackets to distinguish them from durations of notes $n$. 
Example 2. Figure 1 displays some examples of trees with the corresponding notation and rhythm value. ${ }^{5}$

Example 3. The trees in Figure 2 contain the symbol o for the addition of durations as defined in the above semantics. In the tree of Figure 2(a), the duration values of the second leaf (labeled by o) and third leaf (labeled by $n$ ) are summed to express that the second note has a duration of $\frac{1}{2}$ beat. Note that these two leaves are cousin nodes. The idea is the same in Figure 2(b) (here the second note has duration $\frac{1}{3}$ ). In Figure 2(c), two duration values of $\frac{1}{4}$ are also summed, like in Figure 2(a), but here, the obtained duration value of $\frac{1}{2}$ is further divided by 3 . This is expressed by the 3 in the notation, which actually stands for $3: 2$ (3 in the time of 2). Similarly, in the bar Figure 2(d), we have 5 quavers in the time of 3 .

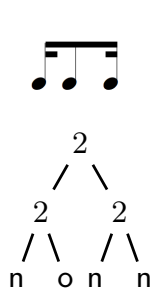

(a) $\frac{1}{4} \frac{1}{2} \frac{1}{4}$

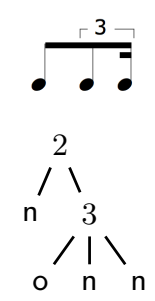

(b) $\frac{1}{2} \frac{1}{3} \frac{1}{6}$
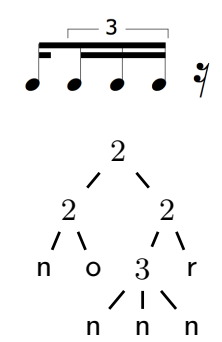

(c) $\frac{1}{4} \frac{1}{6} \frac{1}{6} \frac{1}{6}\left[\frac{1}{4}\right]$

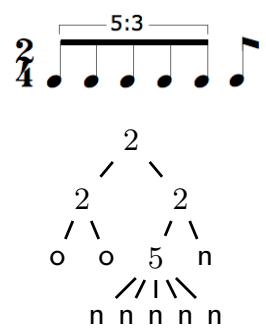

(d) $\frac{3}{10} \frac{3}{10} \frac{3}{10} \frac{3}{10} \frac{3}{10} \frac{1}{2}$

Fig. 2. Example of trees of $\mathcal{T}\left(\Sigma_{\mathrm{rn}}\right)$ : summation with symbol o.

Example 4. Examples of the interpretation of $\mathrm{s}$ and $\mathrm{d}$ in terms of notation are given in Figure 3. In the tree 3(a), a note of duration $\frac{1}{2}$ is dotted, extending its duration to $\frac{3}{4}$. The rhythm value of $3(\mathrm{~b})$ and $3(\mathrm{c})$ is the same as for $3(\mathrm{a})$, but the notation $3(\mathrm{a})$ is more recommended (see [10]).

We define as equivalent the trees representing the same actual rhythm.

Definition 1. Two trees $t_{1}, t_{2} \in \mathcal{T}\left(\Sigma_{\mathrm{rn}}\right)$ are equivalent iff $\operatorname{val}\left(t_{1}\right)=\operatorname{val}\left(t_{2}\right)$.

The tree equivalence relation is denoted $t_{1} \equiv t_{2}$. This notion of equivalence makes it possible to characterize different notations of the same rhythm, like e.g. the trees (a), (b) and (c) in Figure 3.

\footnotetext{
${ }^{5}$ In the examples and figures of this paper, when not specified otherwise with a time signature, we will consider that the duration associated to each of the trees is 1 beat (this duration can also be found by summing the indicated fractional durations).
} 


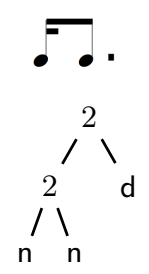

(a) $\frac{1}{4} \frac{3}{4}$

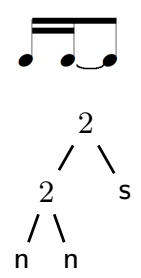

(b) $\frac{1}{4} \frac{3}{4}$

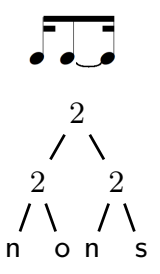

(c) $\frac{1}{4} \frac{3}{4}$

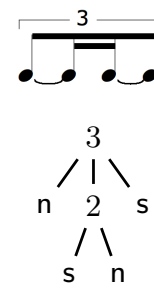

(d) $\frac{1}{2} \frac{1}{2}$

Fig. 3. Trees of $\mathcal{T}\left(\Sigma_{\mathrm{rn}}\right)$ with slurs and dots.

\subsection{Interpretation of Trees into Common Western Notation}

The previous examples showed how the interpretation of trees of $\mathcal{T}\left(\Sigma_{\mathrm{rn}}\right)$ as Common Western Notation is generally straightforward. We describe here some aspects that need particular treatments. We have already said a few words about the case of dots (symbol d), see Example 4. Let us present below another example about tuplets beaming using the symbols of $\overline{\mathbb{P}}$.

Example 5. Figure 4 presents different ways of beaming a sextuplet (see [10]). The three trees are equivalent. In 4(b) and 4(c) the division is respectively bipartite and tripartite. In 4 (a), the division is unclear. The symbols $\overline{2}$ and $\overline{3} \in \overline{\mathbb{P}}$ at the top of the trees 4(b) and 4(c) indicate that there must be only one beam between subtrees (the value one corresponds to the depth of $\overline{2}$ and $\overline{3}$ ). The default rendering, in $4(\mathrm{a})$, with label $3 \in \mathbb{P}$, is that the number of beams between subtrees is the same as the number of beams in subtrees. In Figure 5, similar variants are presented at the scale of a whole bar.

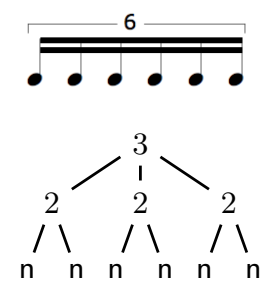

(a) $\frac{1}{6} \frac{1}{6} \frac{1}{6} \frac{1}{6} \frac{1}{6} \frac{1}{6}$

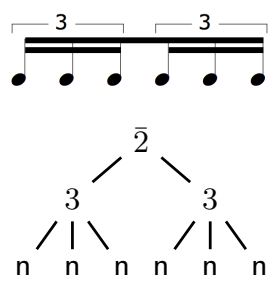

(b) $\frac{1}{6} \frac{1}{6} \frac{1}{6} \frac{1}{6} \frac{1}{6} \frac{1}{6}$

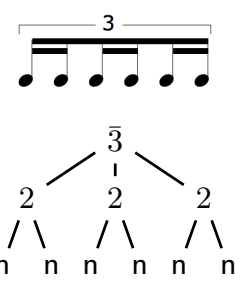

(c) $\frac{1}{6} \frac{1}{6} \frac{1}{6} \frac{1}{6} \frac{1}{6} \frac{1}{6}$

Fig. 4. Tuplet beaming with symbols of $\overline{\mathbb{P}}$ (one beat).

At this point, let us make a few remarks about the above tree semantics.

1) In the tree of Figure 3(a), the dot symbol d labels a leaf node of duration $\frac{1}{2}$, which comes after another leaf node labelled by $\mathrm{n}$ and of duration $\frac{1}{4}$. This may 


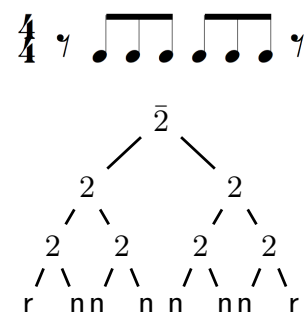

(a) $\left[\frac{1}{2}\right] \frac{1}{2} \frac{1}{2} \frac{1}{2} \frac{1}{2} \frac{1}{2} \frac{1}{2}\left[\frac{1}{2}\right]$

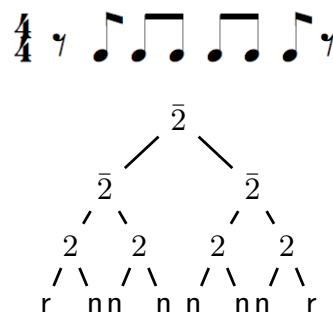

(b) $\left[\frac{1}{2}\right] \frac{1}{2} \frac{1}{2} \frac{1}{2} \frac{1}{2} \frac{1}{2} \frac{1}{2}\left[\frac{1}{2}\right]$

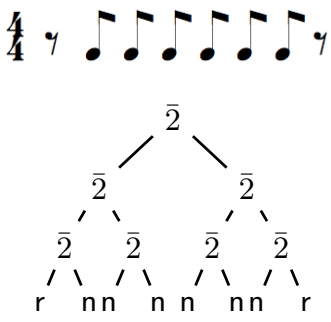

(c) $\left[\frac{1}{2}\right] \frac{1}{2} \frac{1}{2} \frac{1}{2} \frac{1}{2} \frac{1}{2} \frac{1}{2}\left[\frac{1}{2}\right]$

Fig. 5. Tuplet beaming with symbols of $\overline{\mathbb{P}}$ (one bar).

seem counter-intuitive with respect to standard rhythm notation: we could expect the node of duration $\frac{1}{2}$ to be labeled by $n$ and the node of duration $\frac{1}{4}$ to be labeled by $\mathrm{d}$. However in our tree semantics, we have chosen to always represent rooted or tied notes by a $\mathrm{n}$ followed by some $\mathrm{s}$ or $\mathrm{d}$, to avoid ambiguities.

2) Labels d must be used with care for ensuring a correct interpretation into notation. Section 2.3 will discuss some constraints to be satisfied for this sake.

3) The interpretation of the slur symbol $s$ and the dot symbol $d$ is the same regarding durations. This is also the case of $p \in \mathbb{P}$ and $\bar{p} \in \overline{\mathbb{P}}$. The symbols $\mathrm{d}$ and $\bar{p} \in \bar{P}$ have been introduced only to give notation-related information, and the choice of one symbol over the other equivalent will be only dictated by notation preferences (see also Section 2.3).

4) The symbols of $\mathbb{P}$ and $\overline{\mathbb{P}}$ are somehow redundant with the tree structure, since every inner node is labeled with its degree. This technical facility however allowed us to define our trees in the algebra $\mathcal{T}\left(\Sigma_{\mathrm{rn}}\right)$ over a ranked signature $\Sigma_{\mathrm{rn}}$.

\subsection{Syntactical Restrictions and Tree Automata}

In general, several notations can be associated to the same rhythmic value, and the preferences regarding the notation details may depend on varied factors like the metre, usage, or personal preferences of the author. For instance, we have seen in Example 4 (Figure 3) that the dot symbol d produces the same rhythm value as the slur symbol s, but different notations. Also, separating innermost beams by using $\bar{p} \in \overline{\mathbb{P}}$ instead of $p \in \mathbb{P}$, like in Figures 4(b) and 4(c) and Figure 5 , can be useful to reflect the correct subdivisions of a tuplet (following the metre) or indicate accentuations, see [10].

Some constraints regarding notation details can be expressed using tree automata (TAs) over $\Sigma_{\mathrm{rn}}$. TAs in this case correspond to "style files" for rhythm notation.

The first and most important TA that we need in this context is the one characterizing trees with correct interpretation as notation. For instance, following the above remark (2), one can build a TA checking that the d symbols are well placed. This TA recognizes the set of trees of $\mathcal{T}\left(\Sigma_{\mathrm{rn}}\right)$ where a symbol d 
can only occur in a pattern of the form $2(2(x, \mathrm{n}), \mathrm{d})$ or $2(\mathrm{n}, 2(\mathrm{~d}, x))$ is a regular language. We can also extend to double dots by also allowing patterns of the form $2(2(2(x, \mathrm{n}), \mathrm{d}), \mathrm{d})$ or $2(\mathrm{n}, 2(\mathrm{~d}, 2(\mathrm{~d}, x)))$.

Moreover, the compositional properties of TAs make it possible to combine (by union, intersection, complementation) arbitrarily different notation constraints expressed as TAs. For instance, in a binary metre, there exists a TA that allows beaming as in Figure 4(b) and forbids beaming as in Figure 4(c).

\section{Rewrite Rules}

We define a set of rewrite rules on trees, which do not change the rhythmic values (i.e. the actual rhythm), but may change its notation. These rules can therefore be used to produce equivalent notations of a same rhythm. The set of rewrite rules over $\Sigma_{\mathrm{rn}}$ defined in this section will be called $\mathcal{R}_{\mathrm{rn}}$.

\subsection{Normalization Rules}

The following rules reflect the semantical equivalence between dots and slurs (Example 4),

$$
d \rightarrow s
$$

and between symbols of $\overline{\mathbb{P}}$ and their counterpart of $\mathbb{P}$ (Example 5).

$$
\bar{p}\left(x_{1}, \ldots, x_{p}\right) \rightarrow p\left(x_{1}, \ldots, x_{p}\right) \quad p \in \mathbb{P}
$$

Addition of rests. Unlike notes, successive rests are always summed up implicitly. Following this principle, we can decide to merge subdivisions of rests, with standard rewrite rules of the form $2(r, r) \rightarrow r, 3(r, r, r) \rightarrow r$, etc., which are generalized into

$$
p(\underbrace{\mathrm{r}, \ldots, \mathrm{r}}_{p}) \rightarrow \mathrm{r} \quad p \in \mathbb{P}
$$

The use of slurs is useless with rests, hence we have also this rule with cousin patterns of length 2

$$
r ; s \rightarrow r ; r
$$

Similarly, the following rule complies with the semantics of o,

$$
0 ; r \rightarrow r ; r
$$

Normalization of $\mathbf{s}$. According to the semantics of symbols of $\mathbb{P}$, we can simplify fully tied tuplets with standard rewrite rules: $2(\mathrm{~s}, \mathrm{~s}) \rightarrow \mathrm{s}, 3(\mathrm{~s}, \mathrm{~s}, \mathrm{~s}) \rightarrow \mathrm{s}$, etc., generalized into

$$
p(\mathrm{~s}, \ldots, \mathrm{s}) \rightarrow \mathrm{s} \quad p \in \mathbb{P}
$$

We have also $2(\mathrm{n}, \mathrm{s}) \rightarrow \mathrm{n}, 3(\mathrm{n}, \mathrm{s}, \mathrm{s}) \rightarrow \mathrm{n}$, etc., generalized into

$$
p(\mathrm{n}, \mathrm{s}, \ldots, \mathrm{s}) \rightarrow \mathrm{n} \quad p \in \mathbb{P}
$$


Normalization of $\mathrm{o}$. The following rule replaces o by s when possible.

$$
\mathrm{o} ; \mathrm{s} \rightarrow \mathrm{s} ; \mathrm{s}
$$

The semantics presented in Section 2.1 make it possible to sum up the durations corresponding to cousin nodes labeled by $\mathrm{o}$, and then subdivide the duration obtained by this sum. Following this principle, we can sometimes simplify a pattern beginning with a sequence of o's, according to the value of the sum and the number of subdivision. The base case is the subdivision by 1 , corresponding to the atomic note $\mathrm{n}$

$$
\mathrm{o} ; \mathrm{n} \rightarrow \mathrm{n} ; \mathrm{s}
$$

For a subdivision by 2 , we have the following rewrite rules with variables: $\mathrm{o} ; 2\left(x_{1}, x_{2}\right) \rightarrow x_{1} ; x_{2}$, o; $;$; $; 2\left(x_{1}, x_{2}\right) \rightarrow \mathrm{o} ; x_{1} ; \mathrm{o} ; x_{2}$, and so on for each multiple of 2 . For a subdivision by 3 , we have $\mathrm{o} ; \mathrm{o} ; 3\left(x_{1}, x_{2}, x_{3}\right) \rightarrow x_{1} ; x_{2} ; x_{3}$ etc. The general form of the expected transformations is

$$
\underbrace{\mathrm{o} ; \ldots ; \mathrm{o}}_{k p-1} ; p\left(x_{1}, \ldots, x_{p}\right) \rightarrow \underbrace{\mathrm{o} ; \ldots ; 0}_{k-1} ; x_{1} ; \underbrace{\mathrm{o} ; \ldots ; \mathrm{o}}_{k-1} ; x_{2} ; \ldots ; \underbrace{\mathrm{o} ; \ldots ; \mathrm{o} ; x_{p}}_{k-1}
$$

The equation (10) represents a non-bounded number of rules (one for each value of $k$ ). It can be simulated in a finite number of rewrite steps, using auxiliary symbols (which cannot be presented here due to space restrictions).

Example 6. Figure 6 presents a rewrite sequence from the tree in Figure 3(c) into 3(b), and from 3(a) also into 3(b). The nodes of application of rewrite rules are marked by circles, and rewrite rules are indicated. This shows that using the above rewrite rules, we can explore the equivalent trees of Figure 3.

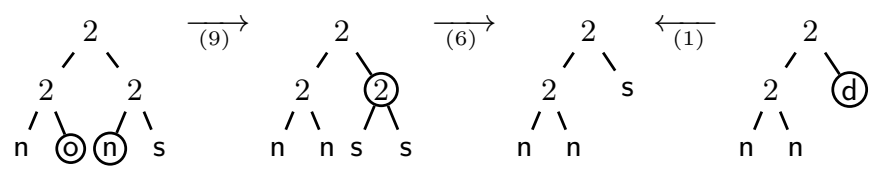

Fig. 6. Rewrite sequences starting from the trees of Figure 3(c) and 3(a). The applied rewriting rule is between parenthesis.

\subsection{Subdivision Equivalence}

Finally, we propose standard rewrite rules for redefining subdivisions, such as

$$
\begin{aligned}
2\left(x_{1}, x_{2}\right) & \rightarrow 3\left(2(\mathrm{o}, \mathrm{o}), 2\left(x_{1}, \mathrm{o}\right), 2\left(\mathrm{o}, x_{2}\right)\right) \\
2\left(x_{1}, x_{2}\right) & \rightarrow 5\left(2(\mathrm{o}, \mathrm{o}), 2(\mathrm{o}, \mathrm{o}), 2\left(x_{1}, \mathrm{o}\right), 2(\mathrm{o}, \mathrm{o}), 2\left(\mathrm{o}, x_{2}\right)\right) \\
3\left(x_{1}, x_{2}, x_{3}\right) & \rightarrow 2\left(3\left(\mathrm{o}, x_{1}, \mathrm{o}\right), 3\left(x_{2}, \mathrm{o}, x_{3}\right)\right) \ldots
\end{aligned}
$$


The general form of these rules is

$$
p\left(x_{1}, \ldots, x_{p}\right) \rightarrow p^{\prime}\left(p\left(u_{1,1}, \ldots, u_{1, p}\right), \ldots, p\left(u_{p^{\prime}, 1}, \ldots, u_{p^{\prime}, p}\right)\right)
$$

where $p, p^{\prime} \in \mathbb{P}, p \neq p^{\prime}$, for all $1 \leq i \leq p^{\prime}, 1 \leq j \leq p, u_{i, j} \in\left\{\mathrm{o}, x_{1}, \ldots, x_{p}\right\}$ and the sequence $u_{1,1}, \ldots, u_{1, p}, \ldots, u_{p^{\prime}, 1}, \ldots, u_{p^{\prime}, p}$ has the form $\underbrace{\mathrm{o}, \ldots, \mathrm{o}, x_{1}}_{p^{\prime}}, \ldots, \underbrace{\mathrm{o}, \ldots, \mathrm{o}, x_{p}}_{p^{\prime}}$.

Example 7. Applying the above rules to the tree of Figure 3(d), we obtain the rewrite sequence depicted in Figure 7 . The result is a simpler tree representing the same durations.

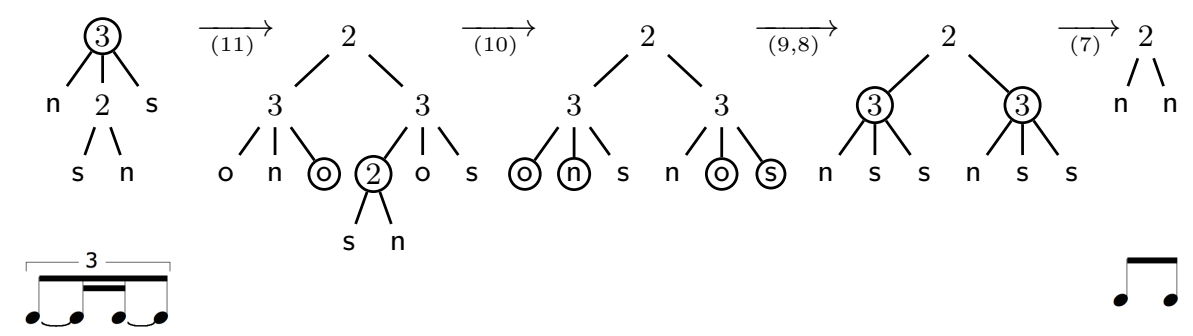

Fig. 7. Rewrite sequence starting from the tree in Figure 3(d).

\section{Properties}

We show that the rewrite rules of $\mathcal{R}_{\mathrm{rn}}$ are correct, in the sense that they preserve rhythmic values of trees, and complete, in the sense that given a tree $t$, it is possible to reach all trees equivalent to $t$ using the rewriting rules of $\mathcal{R}_{\mathrm{rn}}$.

For these properties to hold, we need to consider the following restrictions. A node $\nu$ in a tree $t \in \mathcal{T}\left(\Sigma_{\mathrm{rn}}\right)$ is called dandling if it is labeled by $\mathrm{o}$ and it is not the previous cousin of a node in $\operatorname{dom}(t)$. A tree $t \in \mathcal{T}\left(\Sigma_{\mathrm{rn}} \cup \Theta_{\mathrm{rn}}\right)$ is called o-balanced if for all successive cousins $\nu, \nu^{\prime} \in \operatorname{dom}(t)$ the multisets of labels on the two paths from $\nu$ and $\nu^{\prime}$ up to the root of $t$ are equal. Intuitively, it means that successive cousin nodes labeled by o represent the same duration. A tree $t \in \mathcal{T}\left(\Sigma_{\mathrm{rn}}\right)$ is well-formed iff it is o-balanced and without dandling nodes.

Example 8. All the trees of Figure 3 are well-formed.

The tree of Figure 3(c) can be rewritten into the tree $2(2(n, o), n)$ by rule $(7)$. The latter tree is however not well-formed because of the dandling o-node.

Proposition 1. For all well-formed trees $t_{1}, t_{2} \in \mathcal{T}\left(\Sigma_{\mathrm{rn}}\right), t_{1} \equiv t_{2}$ iff $t_{1} \underset{\mathcal{R}_{\mathrm{r}}}{\stackrel{*}{\longrightarrow}} t_{2}$. 
Let us sketch the proof of Proposition 1. We show by a case analysis that for each rewrite rule $\ell_{1} ; \ldots ; \ell_{n} \rightarrow r_{1} ; \ldots ; r_{n} \in \mathcal{R}_{\mathrm{rn}}$, and for each substitution $\sigma$ grounding for the rule (i.e. such that $\sigma\left(\ell_{1}\right), \ldots, \sigma\left(\ell_{n}\right), \sigma\left(r_{1}\right), \ldots, \sigma\left(r_{n}\right)$ do not contain variables), $\operatorname{val}\left(\sigma\left(\ell_{1}\right) ; \ldots ; \sigma\left(\ell_{n}\right)\right)=\operatorname{val}\left(\sigma\left(r_{1}\right) ; \ldots ; \sigma\left(r_{n}\right)\right)$. The if direction of Proposition 1 then follows from a lifting of this result to the application of contexts, in order to consider rewriting at inner nodes (not only the root node). The proof of the only if direction works by structural induction on $t_{1}$ and $t_{2}$.

We can use the result of Proposition 1 in order to explore rhythm notations equivalent to a given tree, for instance for simplification like in Figures 6 and 7 . In our context, it is reasonable to assume a bounded depth for trees. By Kruskal lemma, it follows that the number of trees to consider is finite.

\section{Conclusion}

The choice of a representation determines the range of possible operations on a given musical structure, and thereby has a significant influence on compositional and analytical processes (see $[11,14]$ for examples in the domain of rhythm structures). In this paper we proposed a formal tree-structured representation for rhythm inspired by previous theoretical models for term rewriting. Based on this representation, tree rewriting can be seen as a mean for transforming rhythms in composition or analysis processes. In a context of computer-aided composition for instance, this approach can make it possible to suggest to a user various notations of the same rhythmic value, with different complexities. Similarly, the rewrite sequence of Figure 7 can be seen as a notation simplification for a given rhythm. An important problem in the confluence of the defined rewrite relation, i.e. whether different rewriting from a single tree will eventually converge to a unique canonical form. For a quantitative approach, it is possible to use standard complexity measures for trees (involving depth, number of symbols etc.). We can therefore imagine that this framework being used as a support for rhythm quantification processes [1] in computer-aided composition environments like OpenMusic.

The tree format that we are proposing has similarities with the Patchwork/OpenMusic Rhythmic Tree (RT) formalism [2]. ${ }^{6}$ Still, these two formats present a number of important differences. While RTs represent durations with integers labeling nodes (the subdivision ratios), our representation only uses the tree structure (i.e., the labels in $\mathbb{P}$ are not formally needed) and labels in a finite (and small) set for leaves. This specificity makes the representation more amenable to purely syntactical processing, when RT processing needs arithmetic. Trees of $\mathcal{T}\left(\Sigma_{\mathrm{rn}}\right)$ and OM RTs are meant to be complementary, and some functions for converting trees back and forth between these two formats have been implemented. The definition domains of these functions are characterized by TA.

\footnotetext{
${ }^{6}$ A rhythm tree RT is defined as a pair $\langle d, S\rangle$ where $d \in \mathbb{N}$ is a duration and $S=$ $s_{0}, \ldots, s_{n}$ is a sequence of subdivisions where for all $1 \leq i \leq n, s_{i}$ is either a tree or a ratio. Formally, $s_{i} \in \mathbb{N}$ or $s_{i}$ is a $\mathrm{RT}$.
} 
As mentioned in Section 2.3, it is also possible to use a TA to complement the rewriting rules and control the rhythm simplification processes by filtering out rewritten trees that do not correspond to actual notations (e.g. because of misplaced d), or/and restricting the search space to trees corresponding to acceptable or preferred notations. This approach is comparable to the use of schemas for XML data processing.

Note that the symbol $\mathrm{n}$ could be replaced by several symbols encoding pitches in order to represent actual melodies. Similar tree-based encodings have been used in [4] for the search of melodic similarities. Finally, other rewrite rules can be considered, including ones that do not preserve durations or rhythmic values. In this case, tree rewriting could constitute a novel creative approach to rhythm transformation in compositional applications. Another application could be the formalization of summarization of music by pruning trees like in [15].

\section{References}

1. C. Agon, G. Assayag, J. Fineberg, and C. Rueda. Kant: a Critique of Pure Quantification. In Int. Computer Music Conf. Proc. (ICMC), pages 52-59, 1994.

2. C. Agon, K. Haddad, and G. Assayag. Representation and Rendering of Rhythm Structures. In Proc. 2d Int. Conf. on WEB Delivering of Music (CW'02), pages 109-113, IEEE Computer Society, 2002.

3. G. Assayag, C. Rueda, M. Laurson, C. Agon, and O. Delerue. Computer Assisted Composition at Ircam : PatchWork and OpenMusic. Comp. Music J., 23(3), 1999.

4. J. F. Bernabeu, J. Calera-Rubio, J. M. Iñesta, and D. Rizo. Melodic Identification Using Probabilistic Tree Automata. J. of New Music Research, 40(2):93-103, 2011.

5. L. Bigo and A. Spicher. Self-assembly of musical representations in MGS. International Journal of Unconventionnal Computing, 2014.

6. J. Bresson, C. Agon, and G. Assayag. OpenMusic: Visual Programming Environment for Music Composition, Analysis and Research. In Proc. of the 19th ACM international conference on MultiMedia, pages 743-746. ACM, 2011.

7. H. Comon, M. Dauchet, R. Gilleron, F. Jacquemard, C. Löding, D. Lugiez, S. Tison, and M. Tommasi. Tree Automata Techniques and Applications. http://tata. gforge.inria.fr, 2007.

8. N. Dershowitz and J.-P. Jouannaud. Rewrite systems. In Handbook of Theoretical Computer Science, vol. B, pages 243-320. North-Holland, 1990.

9. M. Good. Lessons from the Adoption of MusicXML as an Interchange Standard. In XML conference, 2006.

10. E. Gould. Behind Bars: The Definitive Guide to Music Notation. Faber Music, 2011.

11. K. Haddad. Livre Premier de Motets: The Concept of TimeBlocks in OpenMusic. In The OM Composer's Book 2. Editions Delatour - Ircam, 2008.

12. H. H. Hoos, K. A. Hamel, K. Renz, and K. Jürgen. Representing Score-Level Music Using the GUIDO Music-Notation Format. Computing in Musicology, 12, 2001.

13. F. Lerdahl and R. Jackendoff. A Generative Theory of Tonal Music. MIT Press, Cambridge, 1983.

14. M. Malt. Some Considerations on Brian Ferneyhough's Musical Language Through His Use of CAC. In The OM Composer's Book 2. Editions Delatour - Ircam, 2008.

15. D. Rizo. Symbolic music comparison with tree data structures. PhD thesis, Universidad de Alicante, November 2010. 